\title{
Classification of Algebraic Properties of Chromatic Polynomials
}

\author{
S. Rao ${ }^{1 *}$, V. Rao ${ }^{2}$, and A. Kumar ${ }^{3}$ \\ ${ }^{1}$ Department of Mathematics, Guntur Engineering College, Guntur Dt., A.P, India \\ ${ }^{2}$ Department of Mathematics, Mekelle University Main Campus, Mekelle, Ethiopia \\ ${ }^{3}$ Department of Mathematics, Nizam Institute of Engineering and Technology, \\ Nalgonda (Dt), A.P, India
}

Received 7 October 2012, accepted in final revised form 4 July 2013

\begin{abstract}
This manuscript attempts to introduce the concept of chromatic polynomials of total graphs using Mobius inversion theorem. In fact it studies various algebraic properties of chromatic polynomial using Mobius inversion theorem.
\end{abstract}

Keywords: Bond lattice; Chromatic polynomial; Mobius function; Poset.

(C) 2013 JSR Publications. ISSN: 2070-0237 (Print); 2070-0245 (Online). All rights reserved.

doi: http://dx.doi.org/10.3329/jsr.v5i3.11634 J. Sci. Res. 5 (3), 469-477 (2013)

\section{Introduction}

Kirchhoff [1] introduced the chromatic polynomial in 1912 as an attempt to prove the four colour theorem. Today we usually define the chromatic polynomial for arbitrary graphs extended by Whithey [2]. After a while Reed [3] studied about the chromatic polynomials. In addition to that Yap [4] added his contribution on total colourings of graphs. As well Meredith [5] discussed about coefficients of chromatic polynomials in his learning. Further Bender and Goldman [6] contributed on the applications of Mobius inversion in combinatorial analysis. Further Erdos and Wilson [7] put in on the chromatic indexes. Moreover, Rao and Rao [8] initiated the chromatic polynomials of total graphs using deletion-contraction algorithm in their recent study. Also Rao et al. [9-11] contributed to 1-quasi and 2-quasi total graphs.

The aim of this manuscript is to advance the notion of chromatic polynomials of total graphs using Mobius inversion theorem.

${ }^{*}$ Corresponding author: rvnrepalle@ rediffmail.com 


\section{Preliminaries and Fundamental Results}

2.1. Definition [12]: A colouring of a graph $G$ is such that the adjacent vertices have different colours is called a proper colouring of the graph.

2.2. Definition [12]: Let $G$ be a simple graph. The chromatic polynomial of $G$ is the number of ways we can achieve a proper colouring on the vertices of $\mathrm{G}$ with the given $\lambda$ colours and it is denoted by $P(G, \lambda)$ or $P_{\mathrm{G}}(\lambda)$.

2.3. Example: If we want to colour the null graph $\mathrm{N}_{4}$ with $\lambda$ colours, we notice that this can be done in $\lambda^{4}$ ways because there are $\lambda$ colour options for each vertex since no vertex is adjacent to another.

2.4. Definition [13]: Let $G$ be any graph .The total graph of $G, T(G)$ is that a graph whose vertex set is $V(G) \cup E(G)$ and in which two vertices are adjacent if and only if they are adjacent are incident in $G$.

2.5. Example: Fig. 1 represents a $(4,3)$-connected graph $G$ and its total graph $T(G)$.

2.6. Theorem [13]: By deleting an edge and contracting the corresponding vertices until it became a null graph which forms a chromatic polynomial known as the deletion contraction algorithm.

2.7. Definition [13]: A partially ordered set $(S, \leq)$ is a pair consisting of a set $S$ and a binary relation ' $\leq$ 'on $S$ that satisfies the following properties.

(1) Reflexive: For all $x \in S, x \leq x$. (2) Anti symmetric: Given any $x, y \in S$, if $x \leq y$ and $y \leq$ $x$ then $x=y$ and (3) Transitive: For all $x, y, z \in S$, if $x \leq y$ and $y \leq z$ then $x \leq z$.

2.8. Example: The set of all natural numbers with usual ordering " $\leq$ " is a partial order set.

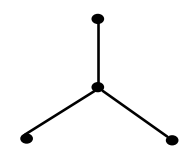

G

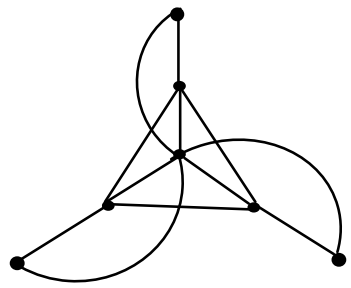

$T(G)$

Fig. 1. A graph $G$ with its total graph $G$.

2.9. Definition [4]: A partially order set with the property that every pair of elements has a greatest lower bound and least upper bound is called a lattice. 
2.10. Example: Let $D_{12}=\{1,2,3,4,6,12\}$ be the set of all divisors of 12 under the relation divides is a lattice.

2.11. Definition [14]: A partition of a set $S$ is defined to be subsets of $S$ which are disjoint and whose union is $S$.Each element of a partition is known as a part.

2.12. Example: Let $S=\{1,2,3,4\}$. Two different partitions of $S$ which are labeled as $P$ and $Q$, let $\mathrm{P}=\{\{1,2\},\{3\},\{4\}\}$ and $Q=\{\{1,2,3\},\{4\}\}$.Two different parts of $P$ are $\{1,2\},\{4\}$.

2.13. Definition [14]: Let $P, Q$ be two partitions of a set $S$, we define a relation between them in which we say that $P$ is finer than $Q$ if every subset, in $P$ is a subset of a subset in $Q$, where $P \neq Q$. We denote this relation by $P \prec Q$.

2.14. Example: From the above example 2.12, $P \prec Q$, since $\{1,2\} \subseteq\{1,2,3\},\{3\}$ $\subseteq\{1,2,3\},\{4\} \subseteq\{4\}$.

2.15. Definition [14]: A bond of a graph $G$ is a partition of its vertices such that all vertices in the same part are connected within the graph. That is they are adjacent or there exists a path between them in the graph that includes only other vertices in the same part. The set of the bonds of a graph form the bond lattice.

2.16. Example: The following figure represents a graph on three vertices and its bond lattice.
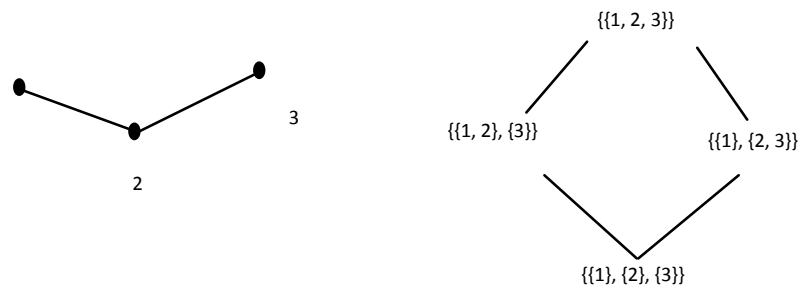

Fig. 2. A graph and its bond lattice.

2.17. Definition [14]: Let $P$ be a partially ordered set. A function $\mu$ defined on $P$ as follows called as the Mobius function. If for any two elements $\mathrm{a}, \mathrm{b}$ of $\mathrm{P}$,

$$
\mu a, b=1 \text { if } a=b ; 0 \text { if } a>b ; \sum_{c: a \leq c<b} \mu(a, c) \text { if } a<b .
$$

\subsection{Mobius inversion theorem}

The principle of Mobius inversion is a significant module of the method of computing chromatic polynomials using bond lattices and the Mobius function. In 1975, Bender and 
Goldman [6] demonstrated Mobius inversion as an over counting-undercounting procedure. The following is Mobius inversion theorem.

Theorem: Let $N_{e} x$ (read "N sub equal to") be a real-valued function defined for all $x$ in a locally finite partially ordered set $S, \leq$ and assume that there is an element $m \in \mathrm{S}$ such that $N_{e} x=0$. Define $N_{a} x \quad$ (read "N sub at least") by $N_{a} x=\sum_{y: y \geq x} N_{e} y$.Thus $N_{e} \quad x=\sum_{y: y \geq x} \mu x, y N_{a} \quad y$

\section{Chromatic Polynomial of Total graph by using Mobius Inversion Theorem}

Rao et al. (2012) established the chromatic polynomial of total graph $T(G)$ of a $(p, q)$ connected graph $G$ (where $p \leq 3$ and $1 \leq q \leq 3$ ) by deletion-contraction method in their recent study. Now we apply the Mobius inversion theorem to total graphs and find the chromatic polynomial of total graph $T(\mathrm{G})$ of a $(p, q)$-connected graph $\mathrm{G}$ restricted for $(2,1)$-connected graph. Fig. 3 represents a $(2,1)-$ connected graph and its total graph and its quasi total graphs.

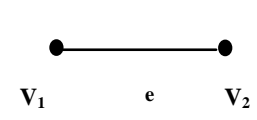

$\mathrm{G}$

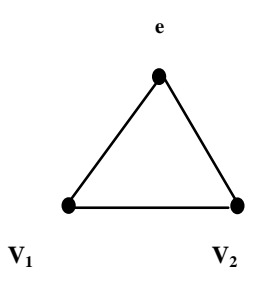

$T(G)$

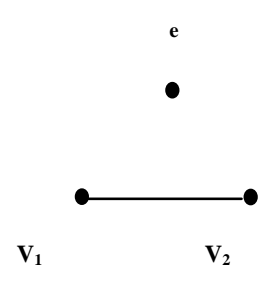

$Q_{1}(G)$

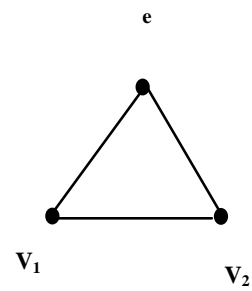

$Q_{2}(G)$

Fig. 3. A graph $G$ of order 2 size 1 with its total graph and its quasi total graphs.

\subsection{Result for chromatic polynomial}

The chromatic polynomial of total graph of a $(p, q)$ - connected graph is $\lambda(\lambda-1)\left[(\lambda-2)^{m+n-2}+\left((\lambda-1)^{m+n-4}\right)\right]$ if $m+n-4>0$, and $\lambda(\lambda-1)\left[(\lambda-2)^{m+n-2}\right]$ if $m+n-4 \leq$ 0 .

Proof. In order to get the chromatic polynomial we need to find the bond lattice of $T(G)$, so that we have an ordering on the bonds of the vertices of $T(G)$. Let the set of vertices of Tree $T(\mathrm{G})$ be $\left\{\mathrm{v}_{1}, \mathrm{v}_{2}, \mathrm{e}\right\}$ as shown in the Fig. 3. Let the partitions be $P_{1=}\left\{\left\{v_{1}\right\},\left\{v_{2}\right\}\right.$, $\{e\}\}, P_{2=\{}\left\{\left\{v_{1}, v_{2}\right\},\{e\}\right\},\left\{\left\{v_{2}, e\right\},\left\{v_{1}\right\}\right\},\left\{\left\{v_{1}, e\right\},\left\{v_{2}\right\}\right\}, P_{3}=\left\{\left\{v_{1}, v_{2}, e\right\}\right\}$.

To find the chromatic polynomial of $T(\mathrm{G})$, we need to calculate $N_{\mathrm{e}}\left\{\left\{v_{1}\right\},\left\{v_{2}\right\},\{e\}\right\}$ the bond $\left\{\left\{v_{1}\right\},\left\{v_{2}\right\},\{e\}\right\}$ represents all colourings in which no two adjacent vertices are the same colour. Let this bond be denoted by $P$, then by Mobius inversion theorem, 
$N_{\mathrm{e}}(\mathrm{P})=\sum_{Q: P \leq Q} \mu(P, Q) N_{a}(Q)$. As a result, for each bond $Q$ in the lattice, we evaluate $\mu(P, Q)$. Let the bond be $P=\left\{\left\{v_{1}\right\},\left\{v_{2}\right\},\{e\}\right\}$. First we have to calculate $\mu(P, Q)$ for all bonds $Q$ in the bond lattice. The first bond in the lowest level has value 1 , while each bond in the second level has value -1. To find the function value of the upper bond $\left\{\left\{\mathrm{v}_{1}, \mathrm{v}_{2}, \mathrm{e}\right\}\right\}$, we simply sum together the values for every other bond and then assign this bond the additive inverse of their value. Hence $\mu\left(P,\left\{\left\{v_{1}, v_{2}, e\right\}\right\}=2\right.$. In our lattice we assign each bond its respected Mobius function value as shown in Fig. 4.

Since all the bonds on the same level have the same number of parts. Thus, for all bonds $Q$ on the same level, $N_{\mathrm{a}}(Q)=\lambda^{\mathrm{i}}$, where $i$ is the common number of parts. Now $N_{\mathrm{a}}\left(P_{1}\right)=\lambda^{3}, N_{\mathrm{a}}\left(P_{2}\right)=\lambda^{2}, N_{\mathrm{a}}\left(P_{3}\right)=\lambda$. We can then sum up the Mobius function values for all the bonds on a particular level and use this value as the coefficient of $\lambda^{\mathrm{i}}$ in our sum.

Hence $\left.N_{\mathrm{e}}(P)=\sum_{Q: P \leq Q} \mu(P, Q) N_{a}(Q)=\lambda^{3}-3 \lambda^{2}+2 \lambda=\lambda(\lambda-1)\left[(\lambda-2)^{1+2-2}\right)\right]=$ $\lambda(\lambda-1)[(\lambda-2)]=\lambda(\lambda-1)\left[(\lambda-2)^{m+n-2}+\left((\lambda-1)^{m+n-4}\right)\right],($ In general $)$.

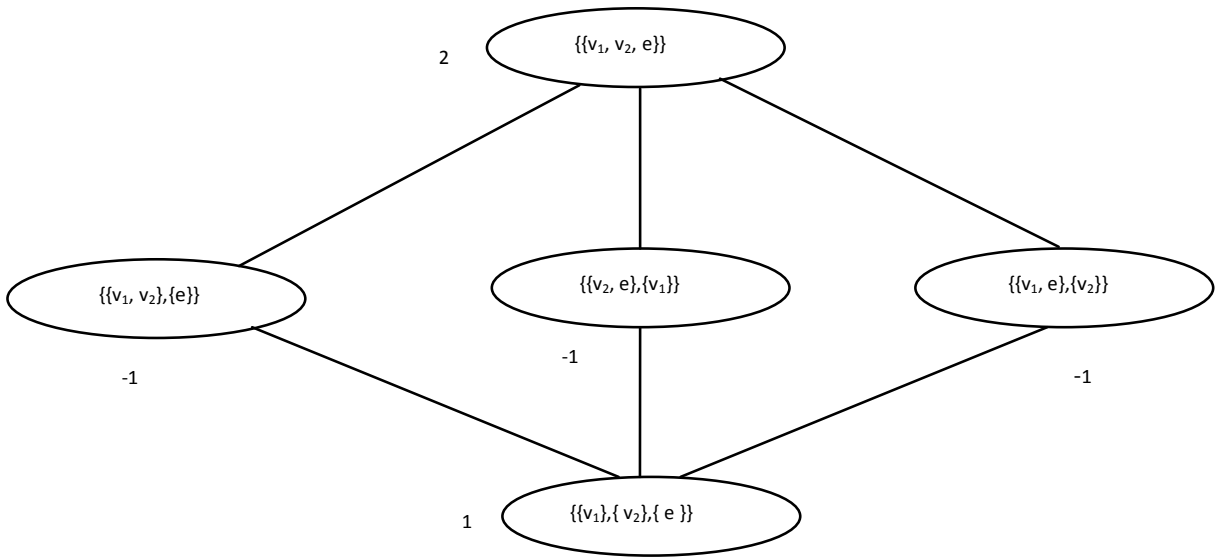

Fig. 4. Bond lattice with attached Mobius function values of a total graph $T(G)$ of a $(2,1)$-connected graph $G$.

3.2. Definition [15]: The 1-quasi total graph of a graph $G, Q_{1}(G)$ is the graph whose vertices set is $V(G) \cup E(G)$ and two vertices are adjacent if and only if they corresponding to two adjacent vertices of $G$ or to two adjacent edges of $G$.

3.3. Definition [15]: The 2-quasi total graph of a graph $G, Q_{2}(G)$ is the graph whose vertices set is $V(G) \cup E(G)$ and two vertices are adjacent if and only if they corresponding to two non adjacent vertices of $G$ or one is a vertex and other is an edge incident with it in G. 
3.4. Example: In Fig. 3 we find the 1-quasi total graph $Q_{1}(G)$ and 2-quasi total graph $Q_{2}(G)$ of a $(2,1)$-connected graph $G$.

\subsection{Result on quasi total graphs}

Here we find the chromatic polynomials of quasi total graphs of a $(2,1)$-connected graph $G$ by using Mobius inversion theorem.

Proof: First we begin with 1- quasi total graph $Q_{1}(G)$ (from Fig. 3)

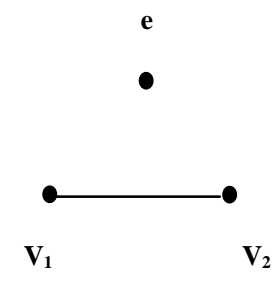

Let the vertices of 1-quasi total graph be $\left\{v_{1}, v_{2}, e\right\}$ and the partitions are $P_{1}=\left\{\left\{v_{1}\right\},\left\{v_{2}\right\},\{e\}\right\}$ and $P_{2}=\left\{\left\{v_{1}, v_{2}\right\},\{e\}\right\}$. We compute the chromatic polynomials of the1quasi total graph given in Fig. 3. In order to achieve this task, we first set up the bond lattice of this graph as shown in Fig. 5. We need to compute $N_{\mathrm{e}}\left(\left\{\left\{v_{1}\right\},\left\{v_{2}\right\},\{e\}\right\}\right)$. As earlier we notice that all the bonds that are found on the same level of the lattice have the same number of parts. Thus, all bonds $Q$ that occurs on the same level, $N_{\mathrm{a}}(Q)=\lambda^{i}$ where $i$ is the common number of parts. Then, we once more sum up the Mobius function values for all the bonds on a particular level and use this resulting value as the co-efficient of $\lambda^{i}$ in our sum. Hence $N_{\mathrm{a}}\left(P_{1}\right)=\lambda^{3}$ and $N_{\mathrm{a}}\left(P_{2}\right)=\lambda^{2}$. The finest bond $P_{1}$ has a value of one while for the coarsest bond has -1 . Now the Mobius function values are $\mu\left(P_{1}, P_{1}\right)=1$ and $\mu\left(P_{1}\right.$, $\left.P_{2}\right)=-1$ (see Fig. 5). Hence by Mobius inversion theorem

$$
\begin{aligned}
& \mathrm{N}_{\mathrm{e}}(\mathrm{P})=\mathrm{N}_{\mathrm{a}}\left(\mathrm{P}_{1}\right) \mu\left(\mathrm{P}_{1}, \mathrm{P}_{1}\right)+\mu\left(\mathrm{P}_{1}, \mathrm{P}_{2}\right) \mathrm{N}_{\mathrm{a}}\left(\mathrm{P}_{2}\right) \\
& =(1) \lambda^{3}+(-1) \lambda^{2}=\lambda^{3}-\lambda^{2}=\left[\lambda^{\mathrm{m}+\mathrm{n}}+(-1)^{\mathrm{m}+\mathrm{n}} \lambda^{\mathrm{m}+\mathrm{n}-1}\right]=\mathrm{P}\left(\mathrm{Q}_{1}(\mathrm{G}), \lambda\right) .(\text { In general })
\end{aligned}
$$

$\mathrm{P}_{2}$

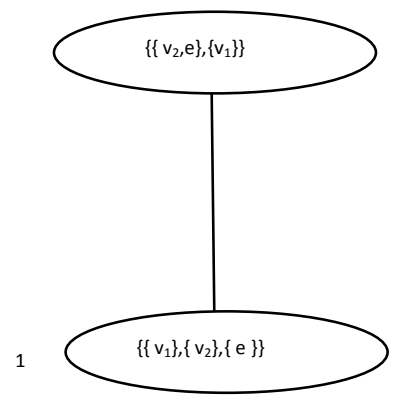

$P_{1}$

Fig. 5. Bond Lattice of $Q_{1}(G)$ with its Mobius function values. 
From Fig. 5, we observed the 2-qasi total graph of $G$ is same as the total graph of $G$, hence the chromatic polynomial of 2-quasi total graph is same as the chromatic polynomial of the total graph $G$ (in particular this case only). Hence

$$
\mathrm{P}\left(\mathrm{Q}_{2}(\mathrm{G}), \lambda\right)=\lambda^{3}-3 \lambda^{2}+2 \lambda \text {. }
$$

\section{Characterizations of Chromatic Polynomials Using Mobius Inversion Theorem}

There are several well known results about the roots and coefficients of the chromatic polynomials being linked to some graph theoretical properties of $G$. We will explore few of these relations using Mobius inversion formula.

4.1. Lemma: The absolute value of coefficient of $\lambda^{\mathrm{n}-1}$ in a chromatic polynomial of $G$ is the number of edges of the graph $G$ of order $n$.

Proof: Let $G$ be finite graph with $n$ vertices. Since to find chromatic polynomial from Mobius inversion theorem, first we find its bond lattice with Mobius function values. Since the second level of any bond lattices is composed of bonds that contain only in edge and singleton vertices. So the number of bonds in the second level of a bond lattice is always the number of edges of the graph. The first level is always composed of only one bond that has Mobius function value of 1 . This value is always finer than every bond on the second level, has a function value of -1 . Because $N_{\mathrm{a}}(b)=\lambda^{\mathrm{n}-1}$ for all bonds $b$ on the second level of any lattices, the coefficient on the $\lambda^{\text {n-1 }}$ terms will always be the negative of the number of edge in the graph.

4.2. Lemma: The sum of the coefficients of any chromatic polynomial is zero except for null graph.

Proof: Since the Mobius function value for each value in the bond lattice of a graph is calculated so that the function value for a particular bond and function values of all finer bonds sum to zero. Particularly the function value for the coarsest bond in the bond lattice is chosen so that the sum of all function values of all bonds in the lattices is zero. Also, we note that plugging $\lambda=1$ into any chromatic polynomial is the same as summing together the entire coefficient. For any graph with at least one edge, this sum will always be zero as such a graph cannot be properly coloured with $\lambda=1$ colours.

4.3. Lemma: The signs on the coefficients of chromatic polynomial are alternate.

Proof: This lemma can be proved by using mathematical induction on levels of the bond lattice of a graph. Suppose our base case is a bond lattice what consists of only two levels. The lower level will consist of only the bond in which each vertex is in a separate part, this bond will always have a Mobius function value of 1 . On the second level, any bond $b$ will have a function value of -1 . Thus chromatic polynomial for the graph described by 
this bond lattice will be $k^{i}-j k^{i-1}$ where $j$ is the number of bonds on the second level of the lattice. We have thus shown that this polynomial has alternating coefficients.

Now, suppose we have a bond lattice that consists of $\mathrm{n}$ levels, where $n>2$, and that our result is true for bond lattice of $n-1$ levels. If we consider only the first $n-1$ levels of our lattice, we know that Mobius function values of the bonds on different level alternative on sign. Now let $b$ be a bond on the $n^{\text {th }}$ level of this lattice. We must show that the Mobius function value of $b$ is not zero that the sign of the function value is different from the sign of the function values for bonds on the $n-1$ level. We know $b$ will not have a function value of zero because we only include bonds in the lattice that correspond to possible sub graphs of the graph we are considering. Now, suppose $b$ is only coarser than one bond on the $n-1$ level denoted by $c$. This is not possible because the Mobius function value of $b$ would have to be zero. This would occur because the function value of $c$ is calculated so that the sum of the function values of all bonds finer than $\mathrm{c}$ and the function value for $c$ is zero. Thus there must least 2 bonds on the $(n-1)$ level that are finer than $b$. Also the sub lattices corresponding to each of these finer bonds must have some sort of overlap; otherwise the function value would again be zero. Because of this overlap, when we sum together the Mobius function value of all bonds finer than $b$, we will find that this sum will have the sign of the bonds on the $n-1$ level, as these values will dominates in the sum. Thus Mobius function value for $b$ must have on opposite sign in order to get the sum back to zero. As a result we have shown that the sign of the Mobius function value in a bond lattice alternative between each level and, consequently that the coefficient of the chromatic polynomials alternative in signs.

\section{Conclusion}

This work extended the concept of chromatic polynomial to total graphs and quasi total graphs using Mobius inversion theorem and established the chromatic polynomials for these graphs. Finally some algebraic properties of chromatic polynomials was established using Mobius inversion theorem.

\section{References}

1. G. D. Birkhoff, Ann. Math. 14, 42 (1912). http://dx.doi.org/10.2307/1967597

2. H. Whitney, Ann. Math. 33, 688 (1932). http://dx.doi.org/10.2307/1968214

3. R. C. Read, J. Combin. Theory 4, 52 (1968). http://dx.doi.org/10.1016/S0021-9800(68)80087-0

4. H. P. Yap, Bull. London Math. Soc. 21, 159 (1968). http://dx.doi.org/10.1112/blms/21.2.159

5. G. H. J. Meredith, J. Combin. Theory B 13, 14 (1972). http://dx.doi.org/10.1016/0095-8956(72)90003-2

6. E. A. Bender and J. R. Goldman, Amer. Math. Monthly 82 (8), 789 (1975). http://dx.doi.org/10.2307/2319793

7. P. Erdos and R. J. Wilson, Combin.Theory Ser. B 23, 255 (1977). http://dx.doi.org/10.1016/0095-8956(77)90039-9

8. R. V. N. S. Rao, J. V. Rao, and D. V. S. R. A. Kumar, Int. J. Math. Computer Appl. Res. 2 (3), 92 (2012). http://tjprc.org/view archives.php?year=2012\&id=45\&jtype=2\&page=3 
9. R. V. N. S. Rao and J. V. Rao, Asian J. Math. Stat. (2012). http://scialert.net/qredirect.php?doi=ajms.2013.52.56\&linkid=pdf

10. R. V. N. S. Rao, J. V. Rao, and D.Srinivasulu, Int. J. Math. Archive 3 (6), 2314 (2012). http://www.ijma.info/index.php/ijma/article/viewFile/1361/766

11. R. V. N. S. Rao and J. V.Rao, Int. J. Math. Archive (7), 2739 (2012). http://www.ijma.info/index.php/ijma/article/view/1473/840

12. F. Harary, Graph Theory (Addison-Wesley, Boston, USA, 1972).

13. D. B. West, Introduction to Graph Theory, $2^{\text {nd }}$ Ed. (Prentice-Hall, India edition, 2003).

14. R. A. Brualadi, Introductory Combinatorics, 3rd ed. (Prentice-Hall, Upper Saddle River, New Jersey, 1999).

15. R. V. N. S. Rao, J. V. Rao, and T. N. Rao, Intern. J. Math. Eng. Sci. ISSN: 2277 (2012). http://www.ijmes.com/uploads/displayVolumeIssue/V-1-I-9-ID-6.pdf 